PROCEEDINGS OF THE

AMERICAN MATHEMATICAL SOCIETY

Volume 139, Number 7, July 2011, Pages 2337-2341

S 0002-9939(2010)10658-5

Article electronically published on December 6, 2010

\title{
ON THE EXPONENT OF THE GROUP OF POINTS OF AN ELLIPTIC CURVE OVER A FINITE FIELD
}

\author{
FRANCESCO PAPPALARDI
}

(Communicated by Ken Ono)

\begin{abstract}
We present a lower bound for the exponent of the group of rational points of an elliptic curve over a finite field. Earlier results considered finite fields $\mathbb{F}_{q} m$ where either $q$ is fixed or $m=1$ and $q$ is prime. Here, we let both $q$ and $m$ vary; our estimate is explicit and does not depend on the elliptic curve.
\end{abstract}

\section{INTRODUCTION}

Let $\mathbb{F}_{q}$ be a finite field with $q=p^{m}$ elements and let $E$ be an elliptic curve defined over $\mathbb{F}_{q}$. It is well known (see for example the book of Washington [7]) that the group of rational point of $E$ over $\mathbb{F}_{q}$ satisfies

$$
E\left(\mathbb{F}_{q}\right) \cong \mathbb{Z}_{n} \times \mathbb{Z}_{n k},
$$

where $n, k \in \mathbb{N}$ are such that $n \mid q-1$. The exponent of $E\left(\mathbb{F}_{q}\right)$ is

$$
\exp \left(E\left(\mathbb{F}_{q}\right)\right)=n k .
$$

The problem of studying $\exp \left(E\left(\mathbb{F}_{q}\right)\right)$ is a natural one and was started by Schoof 6] in 1989. He proved that if $E$ is an elliptic curve over $\mathbb{Q}$ without complex multiplication, then for every prime $p>2$ of good reduction for $E$, one has the estimate

$$
\exp \left(E\left(\mathbb{F}_{p}\right)\right)>C_{E} \sqrt{p} \frac{\log p}{(\log \log p)^{2}},
$$

where $C_{E}>0$ is a constant depending only on $E$.

In 2005, Luca and Shparlinski 4] considered the case when $q$ is fixed and they proved that if $E / \mathbb{F}_{q}$ is ordinary, there exists an effectively computable constant $\vartheta(q)$ depending only on $q$ such that

$$
\exp \left(E\left(\mathbb{F}_{q^{m}}\right)\right)>q^{m / 2+\vartheta(q) m / \log m}
$$

holds for all positive integers $m>1$.

Other lower bounds that hold for families of primes (resp. for families of powers of fixed primes) with density one were proven by Duke in 2] (resp. by Luca and Shparlinski in [4]).

Here we let both $p$ and $m$ vary, and we prove the following:

Received by the editors December 23, 2009 and, in revised form, June 13, 2010 and June 21, 2010

2010 Mathematics Subject Classification. Primary 11G20; Secondary 11G05.

Key words and phrases. Elliptic curves, finite fields.

(C)2010 American Mathematical Society 
Theorem. Let $E$ be any elliptic curve over $\mathbb{F}_{p^{m}}$ where $m \geq 3$. Then either $m=2 r$ is even and

$$
E\left(\mathbb{F}_{p^{2 r}}\right) \cong \mathbb{Z}_{p^{r} \pm 1} \times \mathbb{Z}_{p^{r} \pm 1}
$$

or

$$
\exp \left(E\left(\mathbb{F}_{p^{m}}\right)\right) \geq 2^{-46} p^{m / 2} \frac{m^{1 / 3}}{(\log m)^{8 / 3}(\log \log m)^{1 / 3}} .
$$

Note that the result also applies to supersingular elliptic curves and that it improves on that in (1) for values of $m$ which are small with respect to $p$.

\section{LEMMAS}

The proof is based on estimates for the distance between perfect powers due to Bugeaud. More precisely, we will apply the following result from [1]:

Lemma 1. Let $f \in \mathbb{Z}[X]$ be a monic polynomial of degree $d \geq 2$ without multiple roots. Let $H$ be the maximum of the absolute values of its coefficients and $D$ be its discriminant. Let $a, x, y$, and $m$ be rational integers satisfying $a \neq 0,|y| \geq 2$, $m \geq 2, f(x)=a y^{m}$. Denote by $\log _{2}$ the logarithm in base 2 and write $\log _{*} x$ for $\max \{\log x, 1\}$. The following inequality holds:

$$
m<\max \left\{d \log _{2}(2 H+3), 2^{15(d+6)} d^{7 d}|D|^{3 / 2}(\log |D|)^{3 d}\left(\log _{*}|a|\right)^{2} \log _{*} \log _{*}|a|\right\} .
$$

We need the following elementary lemma:

Lemma 2. If $q$ is a prime power and $E$ is an elliptic curve defined over $\mathbb{F}_{q}$ such that $E\left(\mathbb{F}_{q}\right) \cong \mathbb{Z}_{n} \times \mathbb{Z}_{n k}$, then $q=n^{2} k+n \ell+1$ for some integer $\ell$ that satisfies $|\ell| \leq 2 \sqrt{k}$.

Proof. By the Hasse bound, we can write $n^{2} k=q+1-a_{q}$ for some integer $a_{q}$ that satisfies $a_{q}^{2} \leq 4 q$. Using the Weil pairing one also sees that $q \equiv 1(\bmod n)$. Hence $a_{q}=2+n \ell$ for some integer $\ell$ and $q=n^{2} k+n \ell+1$. Finally

$$
n^{2} \ell^{2}+4 n \ell+4=a_{q}^{2} \leq 4 q=4 n^{2} k+4 n \ell+4,
$$

and the result follows.

We will also need the classical characterizations of the group structures due to Waterhouse (see [7, Theorem 4.3, page 98]) which describes possible cardinalities $\# E\left(\mathbb{F}_{q}\right)$ of the set of $\mathbb{F}_{q}$-rational points of elliptic curves over $\mathbb{F}_{q}$.

Lemma 3. Let $q=p^{m}$ be a power of a prime $p$ and let $N=q+1-a$. There is an elliptic curve $E$ defined over $\mathbb{F}_{q}$ such that $\# E\left(\mathbb{F}_{q}\right)=N$ if and only if $|a| \leq 2 \sqrt{q}$ and a satisfies one of the following:

(i) $\operatorname{gcd}(a, p)=1$;

(ii) $m$ even and $a= \pm 2 \sqrt{q}$;

(iii) $m$ is even, $p \not \equiv 1(\bmod 3)$, and $a= \pm \sqrt{q}$;

(iv) $m$ is odd, $p=2$ or 3 , and $a= \pm p^{(m+1) / 2}$;

(v) $m$ is even, $p \not \equiv 1(\bmod 4)$, and $a=0$;

(vi) $m$ is odd and $a=0$.

For each admissible cardinality, Rück (see Washington [7, Theorem 4.4, page 98]) describes the possible group structures. 
Lemma 4. Let $N$ be an integer that occurs as the order of an elliptic curve over a finite field $\mathbb{F}_{q}$, where $q=p^{m}$ is a power of a prime $p$. Write $N=p^{e} n_{1} n_{2}$ with $p \nmid n_{1} n_{2}$ and $n_{1} \mid n_{2}$ (possibly $n_{1}=1$ ). There is an elliptic curve $E$ over $\mathbb{F}_{q}$ such that

if and only if

$$
E\left(\mathbb{F}_{q}\right) \cong \mathbb{Z}_{p^{e}} \times \mathbb{Z}_{n_{1}} \times \mathbb{Z}_{n_{2}}
$$

(1) $n_{1}=n_{2}$ in case (ii) of Lemma 3;

(2) $n_{1} \mid q-1$ in all other cases of Lemma 3 .

Finally we need the following numerical statement:

Lemma 5. Assume that $\alpha$ and $\beta$ are real numbers with $\alpha>4$ and $\beta \geq 4$. If

$$
\alpha \leq \beta^{3 / 2} \cdot(\log \beta)^{8} \cdot \log \log \beta,
$$

then

$$
\beta \geq \frac{\alpha^{2 / 3}}{(\log \alpha)^{16 / 3}(\log \log \alpha)^{2 / 3}} .
$$

Proof. If $\alpha \geq \beta \geq 4$, then

$$
\beta \geq\left(\frac{\alpha}{(\log \beta)^{8} \log \log \beta}\right)^{2 / 3} \geq\left(\frac{\alpha}{(\log \alpha)^{8} \log \log \alpha}\right)^{2 / 3} .
$$

If $4<\alpha \leq \beta$,

$$
\beta \geq \alpha \geq \frac{\alpha^{2 / 3}}{(\log \alpha)^{16 / 3}(\log \log \alpha)^{2 / 3}} .
$$

\section{Proof of the Theorem}

Assume that $E\left(\mathbb{F}_{p^{m}}\right) \cong \mathbb{Z}_{n} \times \mathbb{Z}_{n k}$. Then, by Lemma 2 we have that

$$
p^{m}=k n^{2}+\ell n+1 \quad \text { for some } \ell \text { with }|\ell| \leq 2 \sqrt{k} .
$$

If $\ell= \pm 2 \sqrt{k}$, then $k$ must be a perfect square, and we write $k=M^{2}$ so that $\ell= \pm 2 M$. Therefore in the above identity we have

$$
p^{m}=(M n \pm 1)^{2},
$$

which implies that $m=2 r$ is even and that $M n=p^{r} \mp 1$. Furthermore, in this case

$$
p^{m}+1-\# E\left(\mathbb{F}_{p^{m}}\right)=\ell n+2= \pm 2 M n+2= \pm 2 p^{m / 2} .
$$

This happens precisely in case (ii) of Lemma 3. Note also that in this case $p \nmid$ $\# E\left(\mathbb{F}_{p^{m}}\right)$. Hence, by case (1) in Lemma 4, we have that $n=n k$ so that $k=1$.

We conclude that if $l= \pm 2 \sqrt{k}$, then $k=1, n=p^{r} \mp 1$ and finally

$$
E\left(\mathbb{F}_{p^{2 r}}\right) \cong \mathbb{Z}_{p^{r} \pm 1} \times \mathbb{Z}_{p^{r} \pm 1}
$$

From now on, we can assume that $|\ell|<2 \sqrt{k}$. We apply Lemma 1 with the following data:

$$
\begin{gathered}
f(X)=X^{2}+\ell X+k, \quad d=2, \quad|D|=4 k-\ell^{2}, \quad H=k, \\
x=k n, \quad y=p \quad \text { and } a=k .
\end{gathered}
$$

Note that since $|\ell|<2 \sqrt{k}$, we have that $D \neq 0$ so that $f$ has two distinct roots. 
From the identity $k p^{m}=(k n)^{2}+\ell(k n)+k$ and from Lemma 1, it follows that

$$
m \leq \max \left\{2 \log _{2}(2 k+3), 2^{134}(4 k)^{3 / 2}(\log 4 k)^{6}\left(\log _{*} k\right)^{2}\left(\log _{*} \log _{*} k\right)\right\} .
$$

Since we can assume that $k \geq 2$, it follows that

$$
m \leq 2^{134}(4 k)^{3 / 2}(\log 4 k)^{8} \log \log 4 k .
$$

If $4 \leq m \leq 2^{136}$, then $m^{1 / 3} /\left(2^{45}(\log m)^{8 / 3}(\log \log m)^{1 / 3}\right)<1 / 4$, and the statement of the Theorem is vacuous since $\exp \left(E\left(\mathbb{F}_{q}\right)\right) \geq \sqrt{q}-1$ for every $q$.

If $m>2^{136}$, we apply Lemma 5 with $\alpha=m / 2^{134}>4$ and $\beta=4 k$, and we obtain

$$
k \geq \frac{1}{4} \cdot \frac{\left(m / 2^{134}\right)^{2 / 3}}{\left(\log _{*} \frac{m}{2^{134}}\right)^{\frac{16}{3}}\left(\log _{*} \log _{*} \frac{m}{2^{134}}\right)^{\frac{2}{3}}} \geq \frac{1}{2^{\frac{274}{3}}} \cdot \frac{m^{2 / 3}}{(\log m)^{16 / 3}(\log \log m)^{2 / 3}},
$$

and so

$$
\exp \left(E\left(\mathbb{F}_{p^{m}}\right)=n k \geq\left(\sqrt{p^{m}}-1\right) \sqrt{k} \geq p^{m / 2} \frac{m^{1 / 3}}{2^{46}(\log m)^{8 / 3}(\log \log m)^{1 / 3}} .\right.
$$

This concludes the proof of the Theorem.

The constant $2^{-46}$ can be slightly improved with a more careful analysis, but this is not too important.

\section{Conclusion}

To construct curves with a small exponent one can consider a recent result of Matomäki in [5] that states that, for any $\epsilon>0$, there exist infinitely many primes $p$ of the form $p=a n^{2}+1$ with $a<p^{1 / 2+\epsilon}$.

Let $p>3$ be one such prime. Since $p+1-a n^{2}=2$ and $p$ is odd, part (i) of Lemma 3 assures that there exists an ordinary elliptic curve $E$ over $\mathbb{F}_{p}$ with $\# E\left(\mathbb{F}_{p}\right)=a n^{2}$ points. Furthermore, since $p \equiv 1 \bmod n$, part (2) of Lemma 4 assures that one can choose $E$ in such a way that

$$
E\left(\mathbb{F}_{p}\right) \cong \mathbb{Z}_{n} \times \mathbb{Z}_{n a}
$$

This implies that there exists an infinite sequence of primes $p$, each with an ordinary elliptic curve $E / \mathbb{F}_{p}$ such that

$$
\exp \left(E\left(\mathbb{F}_{p}\right)\right)=\text { an }<p^{3 / 4+\epsilon} .
$$

One can also consider, for a prime $p$, the identity

$$
p^{3}+1-(p+2)(p-1)^{2}=3 p-1 .
$$

Since $3 p-1$ is coprime to $p$ and $3 p-1 \leq 2 \sqrt{p^{3}}$, part (i) of Lemma 3 can be applied with $q=p^{3}$ and $N=(p+2)(p-1)^{2}$. It follows that there exists an elliptic curve $E$ over $\mathbb{F}_{p^{3}}$ with $\# E\left(\mathbb{F}_{p^{3}}\right)=(p+2)(p-1)^{2}$ points. Furthermore, if $p \equiv 7 \bmod 9$, we can write $N=n_{1} n_{2}$, where $n_{1}=3(p-1)$ and $n_{2}=\frac{(p+2)(p-1)}{3}$. It is clear that $n_{1} \mid n_{2}$ and that $n_{1} \mid p^{3}-1$, so part (2) of Lemma 4 can be applied. It follows that for every prime $p \equiv 7 \bmod 9$, there exists an ordinary elliptic curve over $\mathbb{F}_{p^{3}}$ such that

$$
E\left(\mathbb{F}_{p^{3}}\right) \cong \mathbb{Z}_{3 p-3} \times \mathbb{Z}_{\frac{(p+2)(p-1)}{3}} .
$$

We immediately conclude that there exists a infinite sequence of distinct $q$ with an elliptic curve $E / \mathbb{F}_{q}$ such that

$$
\exp \left(E\left(\mathbb{F}_{q}\right)\right)=\frac{q^{2 / 3}}{3}(1+o(1)) .
$$


This should be compared on one side with Schoof's result in [6] that (assuming $\mathrm{GRH}$ ) if $E$ is an elliptic curve over $\mathbb{Q}$, there exists a constant $c_{E}$ such that $\exp \left(E\left(\mathbb{F}_{p}\right)\right)<c_{E} p^{7 / 8} \log p$ for infinitely many primes $p$ and on another side with Luca, McKee and Shparlinski's results in [3] that there exists an absolute constant $\rho>0$ such that if $E / \mathbb{F}_{q}$ is a fixed elliptic curve, the inequality

$$
\exp \left(E\left(\mathbb{F}_{q^{m}}\right)\right)<q^{m} \exp \left(-m^{\rho / \log \log m}\right)
$$

holds for infinitely many positive integers $m$.

We wonder if, for every $\epsilon>0$, one can construct an infinite family of prime powers $q$, each with an elliptic curve $E / \mathbb{F}_{q}$ such that

$$
E\left(\mathbb{F}_{q}\right) \not \mathbb{Z}_{\sqrt{q} \pm 1} \times \mathbb{Z}_{\sqrt{q} \pm 1}
$$

and

$$
\exp \left(E\left(\mathbb{F}_{q}\right)\right) \ll_{\epsilon} q^{1 / 2+\epsilon}
$$

or if the $2 / 3$ in (2) can be improved.

\section{ACKNOWLEDGEMENTS}

The author would like to thank Bill Banks, Jorge Jimenez Urroz and Igor Shparlinski for some useful conversations.

\section{REFERENCES}

[1] Bugeaud, Yann, Sur la distance entre deux puissances pures. C. R. Acad. Sci. Paris Sér. I Math. 322 (1996), no. 12, 1119-1121. MR.1396651(97i:11030)

[2] Duke, William, Almost all reductions modulo $p$ of an elliptic curve have a large exponent. C. R. Math. Acad. Sci. Paris 337 (2003), no. 11, 689-692. MR2030403 (2005b:11071)

[3] Luca, Florian; McKee, James; Shparlinski, Igor E., Small exponent point groups on elliptic curves. J. Théor. Nombres Bordeaux 18 (2006), no. 2, 471-476. MR2289434 (2008a:11070)

[4] Luca, Florian; Shparlinski, Igor E., On the exponent of the group of points on elliptic curves in extension fields. Int. Math. Res. Not. 2005, no. 23, 1391-1409. MR2152235 (2006h:11072)

[5] Matomäki, Kaisa, $A$ note on primes of the form $p=a q^{2}+1$. Acta Arith. 137 (2009), 133-137. MR2491532 (2009m:11151)

[6] Schoof, ReNÉ, The exponents of the groups of points on the reductions of an elliptic curve. Arithmetic algebraic geometry (Texel, 1989), 325-335, Progr. Math., 89, Birkhäuser Boston, Boston, MA, 1991. MR.1085266 (91j:11043)

[7] Washington, Lawrence C., Elliptic curves. Number theory and cryptography. Second edition. Discrete Mathematics and its Applications (Boca Raton). Chapman \& Hall/CRC, Boca Raton, FL, 2008. MR2404461 (2009b:11101)

Dipartimento di Matematica, Università Roma Tre, Largo San Leonardo Murialdo 1, I-00146, Roma, Italy

E-mail address: pappa@mat.uniroma3.it 